\title{
STRONG APPROXIMATION OF FRACTIONAL SOBOLEV MAPS
}

\author{
PIERRE BOUSQUET, AUGUSTO C. PONCE, AND JEAN VAN SCHAFTINGEN
}

\begin{abstract}
Brezis and Mironescu have announced several years ago that for a compact manifold $N^{n} \subset \mathbb{R}^{\nu}$ and for real numbers $0<s<1$ and $1 \leq p<\infty$, the class $C^{\infty}\left(\bar{Q}^{m} ; N^{n}\right)$ of smooth maps on the cube with values into $N^{n}$ is dense with respect to the strong topology in the Sobolev space $W^{s, p}\left(Q^{m} ; N^{n}\right)$ when the homotopy group $\pi_{\lfloor s p\rfloor}\left(N^{n}\right)$ of order $\lfloor s p\rfloor$ is trivial. The proof of this beautiful result is long and rather involved. Under the additional assumption that $N^{n}$ is $\lfloor s p\rfloor$ simply connected, we give a shorter proof of their result. Our proof for $s p \geq 1$ is based on the existence of a retraction of $\mathbb{R}^{\nu}$ onto $N^{n}$ except for a small subset in the complement of $N^{n}$ and on the Gagliardo-Nirenberg interpolation inequality for maps in $W^{1, q} \cap L^{\infty}$. In contrast, the case $s p<1$ relies on the density of step functions on cubes in $W^{s, p}$.
\end{abstract}

\section{INTRODUCTION}

We address in this paper the problem of density of smooth maps in the fractional Sobolev spaces $W^{s, p}$ with values into manifolds. More precisely, let $0<s<1$ and $1 \leq p<+\infty$, and let $N^{n}$ be a compact manifold of dimension $n$ imbedded in the Euclidean space $\mathbb{R}^{\nu}$. The class of Sobolev maps $W^{s, p}\left(Q^{m} ; N^{n}\right)$ on the unit $m$ dimensional cube $Q^{m}$ with values into $N^{n}$ is defined as the set of measurable maps $u: Q^{m} \rightarrow \mathbb{R}^{\nu}$ such that

$$
u(x) \in N^{n} \quad \text { for a.e. } x \in Q^{m}
$$

having finite Gagliardo seminorm [12,

$$
[u]_{W^{s, p}\left(Q^{m}\right)}=\left(\int_{Q^{m}} \int_{Q^{m}} \frac{|u(x)-u(y)|^{p}}{|x-y|^{m+s p}} \mathrm{~d} x \mathrm{~d} y\right)^{1 / p} .
$$

The following question arises naturally: does $W^{s, p}\left(Q^{m} ; N^{n}\right)$ coincide with the closure of smooth maps $C^{\infty}\left(\bar{Q}^{m} ; N^{n}\right)$ with respect to the distance given by

$$
d_{s, p}(u, v)=\|u-v\|_{L^{p}\left(Q^{m}\right)}+[u-v]_{W^{s, p}\left(Q^{m}\right)} ?
$$

This is indeed the case when $s p \geq m$ :

Proposition 1.1. If $s p \geq m$, then the family of smooth maps $C^{\infty}\left(\bar{Q}^{m} ; N^{n}\right)$ is strongly dense in $W^{s, p}\left(Q^{m} ; N^{n}\right)$.

Here is the sketch of the argument: given $u \in W^{s, p}\left(Q^{m} ; N^{n}\right)$, we consider the convolution $\varphi_{\epsilon} * u$ with a smooth kernel $\varphi_{\epsilon}$. If the range of $\varphi_{\epsilon} * u$ lies in a small tubular neighborhood of $N^{n}$, then we may project $\varphi_{\epsilon} * u$ pointwisely into $N^{n}$. We can always do this for $\epsilon>0$ sufficiently small as long as $s p \geq m$. Indeed, in this case $W^{s, p}\left(Q^{m} ; \mathbb{R}^{\nu}\right)$ imbeds into the space of functions of vanishing mean oscillation $\operatorname{VMO}\left(Q^{m} ; \mathbb{R}^{\nu}\right)$, whence dist $\left(\varphi_{\epsilon} * u, N^{n}\right)$ converges uniformly to $0[9$, Eq. (7)].

2010 Mathematics Subject Classification. 58D15 (46E35, 46T20).

Key words and phrases. Strong density; Sobolev maps; fractional Sobolev spaces; simply connectedness. 
The counterpart of Proposition 1.1 for $W^{1, p}\left(Q^{m} ; N^{n}\right)$ and $p \geq m$ is due to Schoen and Uhlenbeck [25]. The role played by VMO functions in this problem has been first observed by Brezis and Nirenberg 9 .

In the subtler case $s p<m$, the answer to the density problem only depends on the topology of the manifold $N^{n}$ :

Theorem 1. If $s p<m$, then $C^{\infty}\left(\bar{Q}^{m} ; N^{n}\right)$ is strongly dense in $W^{s, p}\left(Q^{m} ; N^{n}\right)$ if and only if $\pi_{\lfloor s p\rfloor}\left(N^{n}\right) \simeq\{0\}$.

We denote by $\lfloor s p\rfloor$ the integral part of $s p$ and for every $\ell \in \mathbb{N}, \pi_{\ell}\left(N^{n}\right)$ is the $\ell$ th homotopy group of $N^{n}$. The topological assumption $\pi_{\lfloor s p\rfloor}\left(N^{n}\right) \simeq\{0\}$ means that every continuous map $f: \mathbb{S}^{\lfloor s p\rfloor} \rightarrow N^{n}$ on the $\lfloor s p\rfloor$ dimensional sphere is homotopic to a constant map. The necessity of this condition has been known for some time [11, Theorem 3; 22, Theorem 4.4; 25, Section 4, Example].

Brezis and Mironescu have announced this beautiful result in a personal communication in April 2003 and a sketch of the proof can be found for instance in [21, pp. 205-206]. The analog of Theorem 1 for $W^{1, p}$ Sobolev maps had been obtained by Bethuel in his seminal paper [2] (see also [14]). Partial results for fractional Sobolev exponents $s$ were known when the manifold $N^{n}$ is a sphere with dimension $n \geq s p\left[11\right.$ ] and also in the setting of trace spaces with $s=1-\frac{1}{p}[3,23]$.

The proof of Theorem 1 is long and quite involved. In this paper we prove the reverse implication of Theorem 1 in the case of $\lfloor s p\rfloor$ simply connected manifolds $N^{n}$. Under this assumption, we give a shorter argument which leads to the following:

Theorem 2. If $s p<m$ and if for every $\ell \in\{0, \ldots,\lfloor s p\rfloor\}$,

$$
\pi_{\ell}\left(N^{n}\right) \simeq\{0\}
$$

then $C^{\infty}\left(\bar{Q}^{m} ; N^{n}\right)$ is strongly dense in $W^{s, p}\left(Q^{m} ; N^{n}\right)$.

This condition has been used by Hajłasz 13 to give a simpler proof of Bethuel's density result for $W^{1, p}$. In [6], we explain how Hajłasz's strategy can be implemented for every Sobolev exponent $s \geq 1$ using some pointwise estimates involving the maximal function operator inspired from the work of Maz'ya and Shaposhnikova 19 .

In order to treat the case $s<1$, we introduce here an additional ingredient based on the density of maps which are smooth except for a small set. The case $s p \geq 1$ is covered by Proposition 2.1 below which relies on a projection argument due to Hardt and Lin [15] (Lemma 2.2 below) and on analytical estimates by Bourgain, Brezis and Mironescu [5]. The case $s p<1$ is based on the density of step functions on cubes (Proposition 3.2 below) inspired by the works of Escobedo [1] and Bourgain, Brezis and Mironescu 4 .

\section{Strong DENSity FOR $\boldsymbol{s} \boldsymbol{p} \geq \mathbf{1}$}

The proof of Theorem 2 for $s p \geq 1$ is based on two main ingredients: (1) when the manifold $N^{n}$ is $\lfloor s p\rfloor$ simply connected, smooth maps are strongly dense in $W^{1, q}\left(Q^{m} ; N^{n}\right)$ for every $1 \leq q<\lfloor s p\rfloor+1$ and (2) locally Lipschitz continuous maps outside a set of dimension $m-\lfloor s p\rfloor-1$ are dense in $W^{s, p}\left(Q^{m} ; N^{n}\right)$.

The proof of the first assertion can be found in [6, 13. Before giving the precise statement of the second assertion, we introduce for $j \in\{0, \ldots, m-2\}$ the class $\mathcal{R}_{j}\left(Q^{m} ; N^{n}\right)$ of maps $u: \bar{Q}^{m} \rightarrow N^{n}$ such that

(i) there exists a finite union of $j$ dimensional submanifolds $T \subset \mathbb{R}^{m}$ such that $u$ is locally Lipschitz continuous in $\bar{Q}^{m} \backslash T$, 
(ii) for almost every $x \in \bar{Q}^{m} \backslash T$,

$$
|D u(x)| \leq \frac{C}{\operatorname{dist}(x, T)},
$$

for some constant $C>0$ depending on $u$.

We observe that for every $1 \leq q<m-j, \mathcal{R}_{j}\left(Q^{m} ; N^{n}\right) \subset W^{1, q}\left(Q^{m} ; N^{n}\right)$, whence by the Gagliardo-Nirenberg interpolation inequality [8, 20, Remark 1], for every $0<s<1$,

$$
\mathcal{R}_{j}\left(Q^{m} ; N^{n}\right) \subset W^{s, \frac{q}{s}}\left(Q^{m} ; N^{n}\right) .
$$

In particular, $\mathcal{R}_{m-\lfloor s p\rfloor-1}\left(Q^{m} ; N^{n}\right)$ is a subset of $W^{s, p}\left(Q^{m} ; N^{n}\right)$.

Assertion (2) above can be stated as follows:

Proposition 2.1. If $1 \leq s p<m$ and $N^{n}$ is $\lfloor s p\rfloor-1$ simply connected, then the class $\mathcal{R}_{m-\lfloor s p\rfloor-1}\left(Q^{m} ; N^{n}\right)$ is strongly dense in $W^{s, p}\left(Q^{m} ; N^{n}\right)$.

The proof of Theorem 1 by Brezis and Mironescu is based on the fact that $\mathcal{R}_{m-\lfloor s p\rfloor-1}\left(Q^{m} ; N^{n}\right)$ is strongly dense in $W^{s, p}\left(Q^{m} ; N^{n}\right)$ for every compact manifold $N^{n}$. This is also known to be the case for every $s \in \mathbb{N}_{*}[2,7$. A previous density result of this type for $\mathbb{S}^{1}$ valued maps in $W^{\frac{1}{2}, 2}$ is due to Rivière [24] (see also [5]).

We temporarily assume Proposition 2.1 and complete the proof of Theorem 2 ,

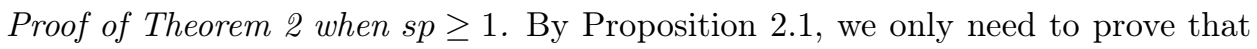
any map $u \in \mathcal{R}_{m-\lfloor s p\rfloor-1}\left(Q^{m} ; N^{n}\right)$ can be approximated in the $W^{s, p}$ norm by smooth maps.

Since $u \in W^{1, q}\left(Q^{m} ; N^{n}\right)$ for every $1 \leq q<\lfloor s p\rfloor+1$, by the topological assumption on the manifold $N^{n}$ there exists a sequence of smooth maps converging to $u$ in $W^{1, q}\left(Q^{m} ; N^{n}\right)$. When $s p>1$, we may take $q=s p$ and by the GagliardoNirenberg interpolation inequality [4, Lemma D.1] the same sequence converges to $u$ in $W^{s, p}\left(Q^{m} ; N^{n}\right)$. The Gagliardo-Nirenberg interpolation inequality fails for $q=1$ in the sense that $W^{1,1} \cap L^{\infty}$ is not continuously imbedded into $W^{s, \frac{1}{s}}$. When $s p=1$ we then take any fixed $1<q<2$ and by the Gagliardo-Nirenberg interpolation inequality $W^{s, p}$ is continuously imbedded in $W^{1, q}$. This implies that the sequence converges to $u$ in $W^{s, p}\left(Q^{m} ; N^{n}\right)$ as before.

We now turn ourselves to the proof of Proposition 2.1. The main geometric ingredient asserts the existence of a retraction from a cube $Q_{R}^{\nu}$ onto $N^{n}$ except for a small set [15, Lemma 6.1]:

Lemma 2.2. Let $\ell \in\{0, \ldots, \nu-2\}$. If $N^{n}$ is $\ell$ simply connected and contained in a cube $Q_{R}^{\nu}$ for some $R>0$, then there exist a closed subset $X \subset Q_{R}^{\nu} \backslash N^{n}$ contained in a finite union of $\nu-\ell-2$ dimensional planes and a locally Lipschitz retraction $\kappa: Q_{R}^{\nu} \backslash X \rightarrow N^{n}$ such that for $x \in Q_{R}^{\nu} \backslash X$,

$$
|D \kappa(x)| \leq \frac{C}{\operatorname{dist}(x, X)},
$$

for some constant $C>0$ depending on $\nu$ and $N^{n}$.

Proof. Let $\mathcal{K}$ be a triangulation of a polyhedral neighborhood $K^{\nu}$ of $N^{n}$ such that $N^{n}$ is a Lipschitz deformation retract of $K^{\nu}$. In particular, $K^{\nu}$ and $N^{n}$ are homotopically equivalent [16, p. 3] and there exists a Lipschitz retraction $h: K^{\nu} \rightarrow$ $N^{n}$. We extend $\mathcal{K}$ as a triangulation of $Q_{R}^{\nu}$ that we denote by $\mathcal{T}$. Since for every $j \in\{0, \ldots, \ell\}$,

$$
\pi_{j}\left(K^{\nu}\right) \simeq \pi_{j}\left(N^{n}\right) \simeq\{0\}
$$


there exists a Lipschitz retraction $g: T^{\ell+1} \cup K^{\nu} \rightarrow K^{\nu}$. Denoting by $\mathcal{L}$ a dual skeleton of $\mathcal{T}$ [26. Chapter 6], let $f:\left(T^{\nu} \backslash L^{\nu-\ell-2}\right) \cup K^{\nu} \rightarrow T^{\ell+1} \cup K^{\nu}$ be a locally Lipschitz retraction such that for every $x \in\left(T^{\nu} \backslash L^{\nu-\ell-2}\right) \cup K^{\nu}$,

$$
|D f(x)| \leq C \frac{1}{\operatorname{dist}\left(x, L^{\nu-\ell-2}\right)} .
$$

The conclusion follows by taking

$$
X:=\overline{L^{\nu-\ell-2} \backslash K^{\nu}} \quad \text { and } \quad \kappa:=h \circ g \circ f .
$$

The next lemma ensures that the approximation we construct in the proof of Proposition 2.1 belongs to a suitable class $\mathcal{R}_{j}$.

Lemma 2.3. Let $\Omega \subset \mathbb{R}^{m}$ be an open set, $v \in C^{\infty}\left(\Omega ; \mathbb{R}^{\nu}\right)$ and let $\lambda \in \mathbb{N}$ be such that $\lambda \leq \min \{m, \nu\}$. If $Y \subset \mathbb{R}^{\nu}$ is a finite union of $\nu-\lambda$ dimensional planes, then for almost every $\xi \in \mathbb{R}^{\nu}$,

(i) the set $v^{-1}(Y+\xi)$ is a finite union of smooth submanifolds of $\mathbb{R}^{m}$ of dimension $m-\lambda$

(ii) for every compact subset $K \subset \Omega$ there exists a constant $C>0$ such that for every $x \in K$,

$$
\operatorname{dist}\left(x, v^{-1}(Y+\xi)\right) \leq C \operatorname{dist}(v(x), Y+\xi) .
$$

Proof. We first assume that $Y$ is a single $\nu-\lambda$ dimensional plane and, without loss of generality,

$$
Y=\left\{0^{\prime}\right\} \times \mathbb{R}^{\nu-\lambda}
$$

with $0^{\prime} \in \mathbb{R}^{\lambda}$. Let $P: \mathbb{R}^{\lambda} \times \mathbb{R}^{\nu-\lambda} \rightarrow \mathbb{R}^{\lambda}$ be the orthogonal projection on the $\lambda$ first coordinates. For every $\xi=\left(\xi^{\prime}, \xi^{\prime \prime}\right) \in \mathbb{R}^{\lambda} \times \mathbb{R}^{\nu-\lambda}$,

$$
v^{-1}(Y+\xi)=v^{-1}\left(Y+\left(\xi^{\prime}, 0^{\prime \prime}\right)\right)=v^{-1}\left(P^{-1}\left(\left\{\xi^{\prime}\right\}\right)\right)=(P \circ v)^{-1}\left(\left\{\xi^{\prime}\right\}\right) .
$$

By Sard's lemma, almost every $\xi^{\prime} \in \mathbb{R}^{\lambda}$ is a regular value of the map $P \circ v$. We deduce in this case that $v^{-1}(Y+\xi)$ is an $m-\lambda$ smooth submanifold of $\Omega$.

We pursue the proof of the estimate in $(i i)$ by assuming that $\xi=0$ and $Y$ is of the form (2.1) where every element of $Y$ is a regular value of $P \circ v$. Given $a \in \Omega$ such that $v(a) \in Y$, the linear transformation $P \circ D v(a)$ is surjective, whence there exist $\delta>0$ with $\overline{B_{\delta}^{m}(a)} \subset \Omega$ and a smooth diffeomorphism $\psi: \overline{B_{\delta}^{m}(a)} \rightarrow \mathbb{R}^{m}$ such that for every $x \in \overline{B_{\delta}^{m}(a)}$,

$$
P \circ v(x)=P \circ D v(a)[\psi(x)] .
$$

This is a consequence of the Inverse function theorem. Indeed, let $\psi_{1}$ be the orthogonal projection in $\mathbb{R}^{m}$ onto ker $P \circ D v(a)$ and let $\psi_{2}=\left(\left.P \circ D v(a)\right|_{(\operatorname{ker} P \circ D v(a))^{\perp}}\right)^{-1} \circ$ $P \circ v$. Then, $D\left(\psi_{1}+\psi_{2}\right)(a)=\operatorname{id}_{\mathbb{R}^{m}}$, whence by the Inverse function theorem the function $\psi=\psi_{1}+\psi_{2}$ is a smooth diffeomorphism in a neighborhood of $a$ and satisfies $P \circ v=P \circ D v(a) \circ \psi$.

It follows from (2.2) that dist $(v(x), Y)=\operatorname{dist}(D v(a)(\psi(x)), Y)$. Denoting by

$$
V=(D v(a))^{-1}(Y)
$$

we observe that for every $y \in B_{\delta}^{m}(a), v(y) \in Y$ if and only if $\psi(y) \in V$. Since $\psi$ is a diffeomorphism, there exist $C_{1}>0$ such that for $x \in B_{\delta}^{m}(a)$,

$$
\operatorname{dist}\left(x, v^{-1}(Y) \cap B_{\delta}^{m}(a)\right) \leq C_{1} \operatorname{dist}\left(\psi(x), V \cap \psi\left(B_{\delta}^{m}(a)\right)\right) .
$$

By the counterpart of $(i i)$ for linear transformations, there exists a constant $C_{2}>0$ such that for every $z \in \mathbb{R}^{m}$,

$$
\operatorname{dist}(z, V) \leq C_{2} \operatorname{dist}(D v(a)[z], Y)
$$


this property can be proved using the linear bijection $\left.R \circ D v(a)\right|_{V^{\perp}}$, where $R$ is the orthogonal projection onto $Y^{\perp}$. Thus, for every $x \in B_{\delta}^{m}(a)$,

$$
\operatorname{dist}(\psi(x), V) \leq C_{2} \operatorname{dist}(D v(a)[\psi(x)], Y)=C_{2} \operatorname{dist}(v(x), Y) .
$$

To conclude the argument, take $0<\underline{\delta} \leq \delta$ such that for every $x \in B_{\underline{\delta}}^{m}(a)$,

$$
\operatorname{dist}\left(x, v^{-1}(Y)\right)=\operatorname{dist}\left(x, v^{-1}(Y) \cap B_{\delta}^{m}(a)\right)
$$

and

$$
\operatorname{dist}(\psi(x), V)=\operatorname{dist}\left(\psi(x), V \cap \psi\left(B_{\delta}^{m}(a)\right)\right) .
$$

We deduce from the above that for $x \in B_{\underline{\delta}}^{m}(a)$,

$$
\operatorname{dist}\left(x, v^{-1}(Y)\right) \leq C_{1} C_{2} \operatorname{dist}(v(x), Y) .
$$

Using a covering argument of $K \cap v^{-1}(Y)$, the conclusion follows when $Y$ is a single $\nu-\lambda$ dimensional plane.

We now assume that $Y$ is a finite union of $\nu-\lambda$ dimensional planes $Y_{1}, \ldots, Y_{j}$. The first assertion is true for almost every $\xi \in \mathbb{R}^{\nu}$. Concerning the second assertion, note that for every $x \in \Omega$ and for every $\xi \in \mathbb{R}^{\nu}$,

$$
\operatorname{dist}\left(x, v^{-1}(Y+\xi)\right)=\min _{i \in\{1, \ldots, j\}} \operatorname{dist}\left(x, v^{-1}\left(Y_{i}+\xi\right)\right)
$$

and

$$
\operatorname{dist}(v(x), Y+\xi)=\min _{i \in\{1, \ldots, j\}} \operatorname{dist}\left(v(x), Y_{i}+\xi\right) .
$$

Let $\xi \in \mathbb{R}^{\nu}$. If the estimate holds for every $Y_{i}$ with some constant $C_{i}^{\prime}>0$, then for every $x \in K$,

$$
\begin{aligned}
\operatorname{dist}\left(x, v^{-1}(Y+\xi)\right) & \leq\left(\max _{i \in\{1, \ldots, j\}} C_{i}^{\prime}\right) \min _{i \in\{1, \ldots, j\}} \operatorname{dist}\left(v(x), Y_{i}+\xi\right) \\
& =\left(\max _{i \in\{1, \ldots, j\}} C_{i}^{\prime}\right) \operatorname{dist}(v(x), Y+\xi) .
\end{aligned}
$$

This concludes the proof of the lemma.

Given a domain $\Omega \subset \mathbb{R}^{m}$ and a measurable function $u: \Omega \rightarrow \mathbb{R}^{\nu}$, we now estimate the convolution function $\varphi_{t} * u$ and its derivative in terms of a fractional derivative of $u$. More precisely, given $0<s<1$ and $1 \leq p<+\infty$, define for $x \in \Omega[20$,

$$
D^{s, p} u(x)=\left(\int_{\Omega} \frac{|u(x)-u(y)|^{p}}{|x-y|^{m+s p}} \mathrm{~d} y\right)^{1 / p} .
$$

We assume that $\varphi: \mathbb{R}^{m} \rightarrow \mathbb{R}$ be a mollifier. In other words,

$$
\varphi \in C_{c}^{\infty}\left(B_{1}^{m}\right), \quad \varphi \geq 0 \text { in } B_{1}^{m} \text { and } \int_{B_{1}^{m}} \varphi=1 .
$$

For every $t>0$, define $\varphi_{t}: \mathbb{R}^{m} \rightarrow \mathbb{R}$ for $h \in \mathbb{R}^{m}$ by

$$
\varphi_{t}(h)=\frac{1}{t^{m}} \varphi\left(\frac{h}{t}\right) .
$$

Using the notation above we have the following:

Lemma 2.4. If $u \in W^{s, p}\left(\Omega ; \mathbb{R}^{\nu}\right)$, then for every $t>0$ and for every $x \in \Omega$ such that dist $(x, \partial \Omega)>t$,

(i) $\left|\varphi_{t} * u(x)-u(x)\right| \leq C t^{s} D^{s, p} u(x)$,

(ii) $\left|D\left(\varphi_{t} * u\right)(x)\right| \leq C^{\prime} t^{-(1-s)} D^{s, p} u(x)$,

for some constants $C>0$ depending on $\varphi$ and $C^{\prime}>0$ depending on D $\varphi$ and $p$. 
Proof. By Jensen's inequality,

$$
\begin{aligned}
\left|\varphi_{t} * u(x)-u(x)\right|^{p} & \leq \int_{\mathbb{R}^{m}} \varphi_{t}(h)|u(x-h)-u(x)|^{p} \mathrm{~d} h \\
& =\int_{\mathbb{R}^{m}} \varphi_{t}(h)|h|^{m+s p} \frac{|u(x-h)-u(x)|^{p}}{|h|^{m+s p}} \mathrm{~d} h .
\end{aligned}
$$

Since $\varphi_{t}$ is supported in $B_{t}^{m}$, for every $h \in \mathbb{R}^{m}, \varphi_{t}(h)|h|^{m+s p} \leq C_{1} t^{s p}$. The first inequality follows.

Next, since $\int_{\mathbb{R}^{m}} D \varphi_{t}=0$,

$$
\left|D\left(\varphi_{t} * u\right)(x)\right| \leq \int_{\mathbb{R}^{m}}\left|D \varphi_{t}(h)\right||u(x-h)-u(x)| \mathrm{d} h .
$$

Since

$$
\int_{\mathbb{R}^{m}}\left|D \varphi_{t}\right| \leq \frac{C_{2}}{t}
$$

by Jensen's inequality,

$$
\begin{aligned}
\left|D\left(\varphi_{t} * u\right)(x)\right|^{p} & \leq \frac{C_{2}^{p-1}}{t^{p-1}} \int_{\mathbb{R}^{m}}\left|D \varphi_{t}(h)\right||u(x-h)-u(x)|^{p} \mathrm{~d} h \\
& =\frac{C_{2}^{p-1}}{t^{p-1}} \int_{\mathbb{R}^{m}}\left|D \varphi_{t}(h)\right||h|^{m+s p} \frac{|u(x-h)-u(x)|^{p}}{|h|^{m+s p}} \mathrm{~d} h .
\end{aligned}
$$

Since for every $h \in \mathbb{R}^{m},\left|D \varphi_{t}(h)\right||h|^{m+s p} \leq C_{3} t^{s p-1}$, the second estimate follows.

If $u \in W^{s, p}\left(\Omega ; \mathbb{R}^{\nu}\right)$ and $\kappa: \mathbb{R}^{\nu} \rightarrow \mathbb{R}^{\nu}$ is Lipschitz continuous, then $\kappa \circ u \in$ $W^{s, p}\left(\Omega ; \mathbb{R}^{\nu}\right)$ and

$$
[\kappa \circ u]_{W^{s, p}(\Omega)} \leq|\kappa|_{\operatorname{Lip}\left(\mathbb{R}^{\nu}\right)}[u]_{W^{s, p}(\Omega)},
$$

where $|\kappa|_{\operatorname{Lip}\left(\mathbb{R}^{\nu}\right)}$ denotes the best Lipschitz constant of $\kappa$. The next lemma gives the continuity of the composition operator $u \mapsto \kappa \circ u$ in $W^{s, p}$ :

Lemma 2.5. Let $\Omega \subset \mathbb{R}^{m}$ be a bounded open set and $u \in W^{s, p}\left(\Omega ; \mathbb{R}^{\nu}\right)$. For every $\epsilon>0$, there exists $\delta>0$ such that if $\kappa: \mathbb{R}^{\nu} \rightarrow \mathbb{R}^{\nu}$ is Lipschitz continuous, $v \in W^{s, p}(\Omega)$ and $\|u-v\|_{W^{s, p}\left(\Omega ; \mathbb{R}^{\nu}\right)} \leq \delta$, then

$$
[\kappa \circ u-\kappa \circ v]_{W^{s, p}(\Omega)} \leq|\kappa|_{\operatorname{Lip}\left(\mathbb{R}^{\nu}\right)} \epsilon .
$$

By a result of Marcus and Mizel [18, Theorem 1] in the scalar case $\nu=1$, the map $u \in W^{1, p}(\Omega ; \mathbb{R}) \mapsto \kappa \circ u \in W^{1, p}(\Omega ; \mathbb{R})$ is continuous. Lemma 2.5 has been proved by Bourgain, Brezis and Mironescu [5, Claim (5.43)]. For the convenience of the reader we present their proof, organized differently.

Proof of Lemma 2.5. For $u, v \in W^{s, p}\left(\Omega ; \mathbb{R}^{\nu}\right)$ and $\kappa: \mathbb{R}^{\nu} \rightarrow \mathbb{R}^{\nu}$, define for $x, y \in \Omega$,

$$
I(x, y)=\frac{|\kappa(u(x))-\kappa(v(x))-\kappa(u(y))+\kappa(v(y))|^{p}}{|x-y|^{m+s p}},
$$

so that

$$
[\kappa \circ u-\kappa \circ v]_{W^{s, p}(\Omega)}=\int_{\Omega} \int_{\Omega} I(x, y) \mathrm{d} x \mathrm{~d} y .
$$


Observe that

$$
\begin{aligned}
I(x, y) & \leq 2^{p-1} \frac{|\kappa(u(x))-\kappa(v(x))|^{p}+|\kappa(u(y))-\kappa(v(y))|^{p}}{|x-y|^{m+s p}} \\
& \leq 2^{p-1}|\kappa|_{\operatorname{Lip}\left(\mathbb{R}^{\nu}\right)}^{p} \frac{|u(x)-v(x)|^{p}+|u(y)-v(y)|^{p}}{|x-y|^{m+s p}}
\end{aligned}
$$

and that

$$
\begin{aligned}
I(x, y) & \leq 2^{p-1} \frac{|\kappa(u(x))-\kappa(u(y))|^{p}+|\kappa(v(x))-\kappa(v(y))|^{p}}{|x-y|^{m+s p}} \\
& \leq 2^{p-1}|\kappa|_{\operatorname{Lip}\left(\mathbb{R}^{\nu}\right)}^{p} \frac{|u(x)-u(y)|^{p}+|v(x)-v(y)|^{p}}{|x-y|^{m+s p}} \\
& \leq C_{1}|\kappa|_{\operatorname{Lip}\left(\mathbb{R}^{\nu}\right)}^{p}\left(\frac{|u(x)-u(y)|^{p}}{|x-y|^{m+s p}}+\frac{|u(x)-v(x)-u(y)+v(y)|^{p}}{|x-y|^{m+s p}}\right) .
\end{aligned}
$$

Given $\epsilon>0$, let

$$
A_{v, \epsilon}=\left\{(x, y) \in \Omega \times \Omega:|u(x)-v(x)|^{p}+|u(y)-v(y)|^{p} \geq \epsilon|x-y|^{m+s p}\right\} .
$$

Using the first upper bound of $I(x, y)$ on the set $(\Omega \times \Omega) \backslash A_{v, \epsilon}$ and the second one on the set $A_{v, \epsilon}$, we get

$$
\begin{aligned}
& {[\kappa \circ u-\kappa \circ v]_{W^{s, p}(\Omega)}^{p}} \\
& \quad \leq|\kappa|_{\operatorname{Lip}\left(\mathbb{R}^{\nu}\right)}^{p}\left(2^{p-1} \epsilon|\Omega|^{2}+C_{1} \iint_{A_{v, \epsilon}} \frac{|u(x)-u(y)|^{p}}{|x-y|^{m+s p}} \mathrm{~d} x \mathrm{~d} y+C_{1}[u-v]_{W^{s, p}(\Omega)}^{p}\right) .
\end{aligned}
$$

Since $u \in W^{s, p}(\Omega)$ and $\left|A_{v, \epsilon}\right| \rightarrow 0$ as $v \rightarrow u$ in $W^{s, p}(\Omega)$, the conclusion follows from the Dominated convergence theorem.

Despite of the estimate (2.3), when $\kappa$ is not affine there is no inequality of the form

$$
[\kappa \circ u-\kappa \circ v]_{W^{s, p}(\Omega)} \leq C|\kappa|_{\operatorname{Lip}\left(\mathbb{R}^{\nu}\right)}[u-v]_{W^{s, p}(\Omega)} .
$$

In fact, the map $u \mapsto \kappa \circ u$ is not even uniformly continuous in $W^{s, p}$. We explain the argument when the domain is the unit cube $Q^{m}$. For this purpose, let $\varphi \in$ $C_{c}^{\infty}\left(Q^{m} ; \mathbb{R}^{\nu}\right)$ and denote by $\bar{\varphi}$ the periodic extension of $\varphi$ to $\mathbb{R}^{m}$. Define for $j \in \mathbb{N}_{*}$,

$$
v_{j}(x)=\bar{\varphi}(j x)
$$

and, for some fixed $\xi \in \mathbb{R}^{\nu}$,

$$
u_{j}(x)=\bar{\varphi}(j x)+\xi
$$

We observe that

$$
\left\|u_{j}-v_{j}\right\|_{W^{s, p}\left(Q^{m}\right)}=\left\|u_{j}-v_{j}\right\|_{L^{p}\left(Q^{m}\right)}=2^{m}|\xi|
$$

whereas

$$
\begin{aligned}
& {\left[\kappa \circ u_{j}-\kappa \circ v_{j}\right]_{W^{s, p}\left(Q^{m}\right)}^{p}} \\
& \quad \geq j^{s p} \int_{Q^{m}} \int_{Q^{m}} \frac{|\kappa(\varphi(x)+\xi)-\kappa(\varphi(x))-\kappa(\varphi(y)+\xi)+\kappa(\varphi(y))|^{p}}{|x-y|^{m+s p}} \mathrm{~d} x \mathrm{~d} y .
\end{aligned}
$$

When $\kappa$ is not affine, there exist $\xi, \tau, \sigma \in \mathbb{R}^{\nu}$ such that

$$
\kappa(\tau+\xi)-\kappa(\tau) \neq \kappa(\sigma+\xi)-\kappa(\sigma) .
$$


Taking $\varphi \in C_{c}^{\infty}\left(Q^{m} ; \mathbb{R}^{\nu}\right)$ for which both sets $\varphi^{-1}(\{\sigma\})$ and $\varphi^{-1}(\{\tau\})$ have positive measure, we have

$$
\int_{Q^{m}} \int_{Q^{m}} \frac{|\kappa(\varphi(x)+\xi)-\kappa(\varphi(x))-\kappa(\varphi(y)+\xi)+\kappa(\varphi(y))|^{p}}{|x-y|^{m+s p}} \mathrm{~d} x \mathrm{~d} y>0 .
$$

As we let $j$ tend to infinity in (2.4), we conclude that $u \mapsto \kappa \circ u$ is not uniformly continuous in $W^{s, p}$.

Proof of Proposition [2.1. Let $u \in W^{s, p}\left(Q^{m} ; N^{n}\right)$. The restrictions to $Q^{m}$ of the maps $u_{\gamma} \in W^{s, p}\left(Q_{1+2 \gamma}^{m} ; N^{n}\right)$ defined for $x \in Q_{1+2 \gamma}^{m}$ by $u_{\gamma}(x)=u(x /(1+2 \gamma))$ converge strongly to $u$ in $W^{s, p}\left(Q^{m} ; N^{n}\right)$ as $\gamma$ tends to 0 . We can thus assume from the beginning that $u \in W^{s, p}\left(Q_{1+2 \gamma}^{m} ; N^{n}\right)$ for some $\gamma>0$.

Let $\kappa: \mathbb{R}^{\nu} \backslash X \rightarrow N^{n}$ be the locally Lipschitz retraction of Lemma 2.2 with $\ell=\lfloor s p\rfloor-1$; we may assume that $\nu \geq \ell+2$. For every $\xi \in \mathbb{R}^{\nu}$, we consider the $\operatorname{map} \kappa_{\xi}: \mathbb{R}^{\nu} \backslash(X+\xi) \rightarrow N^{n}$ defined by

$$
\kappa_{\xi}(x)=\kappa(x-\xi) .
$$

Given a mollifier $\varphi$ (see p. 5 above), the map $\kappa_{\xi} \circ\left(\varphi_{t} * u\right)$ is locally Lipschitz continuous in $Q_{1+\gamma}^{m} \backslash\left(\varphi_{t} * u\right)^{-1}(X+\xi)$. Moreover, by the chain rule and by the pointwise estimate satisfied by $D \kappa$,

$$
\left|D\left[\kappa_{\xi} \circ\left(\varphi_{t} * u\right)\right]\right| \leq C_{1} \frac{\left|D\left(\varphi_{t} * u\right)\right|}{\operatorname{dist}\left(\varphi_{t} * u, X+\xi\right)} .
$$

The set $X$ is contained in a finite union of $\nu-\lfloor s p\rfloor-1$ dimensional planes $Y$ in $\mathbb{R}^{\nu}$. Applying Lemma 2.3 to $v=\varphi_{t} * u \in C^{\infty}\left(Q_{1+\gamma}^{m} ; \mathbb{R}^{\nu}\right)$, we obtain that for every $0<t \leq \gamma$ and for almost every $\xi \in \mathbb{R}^{\nu}$, the set $\left(\varphi_{t} * u\right)^{-1}(X+\xi)$ is contained in a finite union of $m-\lfloor s p\rfloor-1$ dimensional submanifolds,

$$
T=\left(\varphi_{t} * u\right)^{-1}(Y+\xi) .
$$

By 2.5) and the inclusion $X \subset Y$,

$$
\left|D\left[\kappa_{\xi} \circ\left(\varphi_{t} * u\right)\right]\right| \leq C_{2} \frac{1}{\operatorname{dist}\left(\varphi_{t} * u, X+\xi\right)} \leq C_{2} \frac{1}{\operatorname{dist}\left(\varphi_{t} * u, Y+\xi\right)} .
$$

By the second part of Lemma 2.3, we conclude that for $x \in \bar{Q}^{m} \backslash\left(\varphi_{t} * u\right)^{-1}(Y+\xi)$,

$$
\left|D\left[\kappa_{\xi} \circ\left(\varphi_{t} * u\right)\right](x)\right| \leq C_{3} \frac{1}{\operatorname{dist}\left(x,\left(\varphi_{t} * u\right)^{-1}(Y+\xi)\right)}=\frac{C_{3}}{\operatorname{dist}(x, T)} .
$$

In particular, for every $0<t \leq \gamma$ and for almost every $\xi \in \mathbb{R}^{\nu}$, the map $\kappa_{\xi} \circ\left(\varphi_{t} * u\right)$ belongs to $\mathcal{R}_{m-\lfloor s p\rfloor-1}\left(Q^{m} ; N^{n}\right)$.

We proceed using an idea from [5] for $W^{\frac{1}{2}, 2}$ maps with values into the circle $\mathbb{S}^{1}$. Let

$$
\alpha=\frac{1}{4} \operatorname{dist}\left(X, N^{n}\right)
$$

let $\theta: \mathbb{R}^{\nu} \rightarrow \mathbb{R}$ be a Lipschitz continuous function such that

(a) for $\operatorname{dist}(x, X) \leq 2 \alpha, \theta(x)=1$,

(b) for $\operatorname{dist}(x, X) \geq 3 \alpha, \theta(x)=0$,

and let

$$
\bar{\kappa}_{\xi}=(1-\theta) \kappa_{\xi} \quad \text { and } \quad \underline{\kappa}_{\xi}=\theta \kappa_{\xi} .
$$


Since $\kappa_{\xi}=\bar{\kappa}_{\xi}$ on $u\left(Q_{1+2 \gamma}^{m}\right) \subset N^{n}$, we have by the triangle inequality,

$$
\begin{aligned}
& \left\|\kappa_{\xi} \circ\left(\varphi_{t} * u\right)-u\right\|_{W^{s, p}\left(Q^{m}\right)} \\
& \leq\left\|\underline{\kappa}_{\xi} \circ\left(\varphi_{t} * u\right)\right\|_{W^{s, p}\left(Q^{m}\right)}+\left\|\bar{\kappa}_{\xi} \circ\left(\varphi_{t} * u\right)-\bar{\kappa}_{\xi} \circ u\right\|_{W^{s, p}\left(Q^{m}\right)} \\
& +\left\|\kappa_{\xi} \circ u-u\right\|_{W^{s, p}\left(Q^{m}\right)} .
\end{aligned}
$$

Since $\kappa$ is Lipschitz continuous on a neighborhood of $N^{n}$ and $\kappa_{\xi} \circ u=\kappa(u-\xi)$, we have by continuity of the composition operator in $W^{s, p}$ (Lemma 2.5),

$$
\lim _{\xi \rightarrow 0}\left\|\kappa_{\xi} \circ u-u\right\|_{W^{s, p}\left(Q^{m}\right)}=0 .
$$

By Lemma 2.5, as the maps $\bar{\kappa}_{\xi}$ are uniformly Lipschitz continuous and $\varphi_{t} * u$ converges to $u$ in $W^{s, p}\left(Q^{m}\right)$,

$$
\lim _{t \rightarrow 0}\left\|\bar{\kappa}_{\xi} \circ\left(\varphi_{t} * u\right)-\bar{\kappa}_{\xi} \circ u\right\|_{W^{s, p}\left(Q^{m}\right)}=0,
$$

uniformly with respect to $\xi$.

It remains to estimate the first term in the right hand side of (2.6). This is done in the following:

Claim. For every $0<t \leq \gamma$,

$$
\int_{B_{\alpha}^{\nu}}\left\|\underline{\kappa}_{\xi} \circ\left(\varphi_{t} * u\right)\right\|_{W^{s, p}\left(Q^{m}\right)}^{p} \mathrm{~d} \xi \leq C \int_{\left\{\left|\varphi_{t} * u-u\right| \geq \alpha\right\}}\left(D^{s, p} u\right)^{p}
$$

We assume temporarily the claim, and complete the proof of Proposition 2.1. Since $D^{s, p} u \in L^{p}\left(Q^{m}\right)$ and $\varphi_{t} * u$ converges to $u$ in measure as $t$ tends to zero, by the claim we have

$$
\lim _{t \rightarrow 0} \int_{B_{\alpha}^{\nu}}\left\|\underline{\kappa}_{\xi} \circ\left(\varphi_{t} * u\right)\right\|_{W^{s, p}\left(Q^{m}\right)}^{p} \mathrm{~d} \xi=0 .
$$

By the Chebyshev inequality,

$$
\begin{array}{r}
\lim _{t \rightarrow 0}\left|\left\{\xi \in B_{\alpha}^{m}:\left\|\underline{\kappa}_{\xi} \circ\left(\varphi_{t} * u\right)\right\|_{W^{s, p}\left(Q^{m}\right)}^{p} \geq\left(\int_{B_{\alpha}^{m}}\left\|\underline{\kappa}_{\zeta} \circ\left(\varphi_{t} * u\right)\right\|_{W^{s, p}\left(Q^{m}\right)}^{p} \mathrm{~d} \zeta\right)^{\frac{1}{2}}\right\}\right| \\
=0 .
\end{array}
$$

Thus, for every $0<t \leq \gamma$, there exists $\xi_{t} \in B_{\alpha}^{m}$ such that $\lim _{t \rightarrow 0} \xi_{t}=0$ and

$$
\lim _{t \rightarrow 0}\left\|\underline{\kappa}_{\xi_{t}} \circ\left(\varphi_{t} * u\right)\right\|_{W^{s, p}\left(Q^{m}\right)}=0 .
$$

We conclude from (2.6), (2.7) and (2.8) that

$$
\lim _{t \rightarrow 0}\left\|\kappa_{\xi_{t}} \circ\left(\varphi_{t} * u\right)-u\right\|_{W^{s, p}\left(Q^{m}\right)}=0 .
$$

This gives the conclusion of Proposition 2.1

It remains to establish the claim:

Proof of the claim. Let $1<q<p<r$ be such that

$$
\frac{1}{p}=\frac{1-s}{r}+\frac{s}{q}
$$

By the Gagliardo-Nirenberg interpolation inequality,

(2.10) $\left\|\underline{\kappa}_{\xi} \circ\left(\varphi_{t} * u\right)\right\|_{W^{s, p}\left(Q^{m}\right)} \leq C_{4}\left\|\underline{\kappa}_{\xi} \circ\left(\varphi_{t} * u\right)\right\|_{L^{r}\left(Q^{m}\right)}^{1-s}\left\|\underline{\kappa}_{\xi} \circ\left(\varphi_{t} * u\right)\right\|_{W^{1, q}\left(Q^{m}\right)}^{s}$.

As $N^{n}$ is compact, we observe that the functions $\underline{\kappa}_{\xi} \circ\left(\varphi_{t} * u\right)$ are uniformly bounded and supported on the set $\left\{\operatorname{dist}\left(\varphi_{t} * u, X\right) \leq 3 \alpha\right\}$. Moreover,

$$
\left\{\operatorname{dist}\left(\varphi_{t} * u, X\right) \leq 3 \alpha\right\} \subset\left\{\left|\varphi_{t} * u-u\right| \geq \alpha\right\} \text {. }
$$


Thus,

$$
\left\|\underline{\kappa}_{\xi} \circ\left(\varphi_{t} * u\right)\right\|_{L^{r}\left(Q^{m}\right)} \leq C_{4}\left|\left\{\left|\varphi_{t} * u-u\right| \geq \alpha\right\}\right|^{\frac{1}{r}} .
$$

Next, by the Leibniz rule and by (2.5),

$$
\begin{aligned}
\left|D\left(\underline{\kappa}_{\xi} \circ\left(\varphi_{t} * u\right)\right)\right| & \leq\left(\left|D \theta\left(\varphi_{t} * u\right)\right|\left|\kappa_{\xi}\left(\varphi_{t} * u\right)\right|+\left|\theta\left(\varphi_{t} * u\right)\right|\left|D \kappa_{\xi}\left(\varphi_{t} * u\right)\right|\right)\left|D\left(\varphi_{t} * u\right)\right| \\
& \leq C_{5}\left(1+\frac{1}{\operatorname{dist}\left(\varphi_{t} * u, X+\xi\right)}\right)\left|D\left(\varphi_{t} * u\right)\right| .
\end{aligned}
$$

Since the functions $D\left(\underline{\kappa}_{\xi} \circ\left(\varphi_{t} * u\right)\right)$ are also supported in the set $\left\{\left|\varphi_{t} * u-u\right| \geq \alpha\right\}$, we get

$$
\begin{aligned}
& \left\|\underline{\kappa}_{\xi} \circ\left(\varphi_{t} * u\right)\right\|_{W^{1, q}\left(Q^{m}\right)}^{q} \\
& \leq C_{6} \int_{\left\{\left|\varphi_{t} * u-u\right| \geq \alpha\right\}}\left[1+\left(1+\frac{1}{\operatorname{dist}\left(\varphi_{t} * u, X+\xi\right)^{q}}\right)\left|D\left(\varphi_{t} * u\right)\right|^{q}\right] .
\end{aligned}
$$

For

$$
q \geq s p
$$

we have by Hölder's inequality and by Fubini's theorem,

$$
\begin{aligned}
& \int_{B_{\alpha}^{\nu}}\left\|\underline{\kappa}_{\xi} \circ\left(\varphi_{t} * u\right)\right\|_{W^{1, q}\left(Q^{m}\right)}^{s p} \mathrm{~d} \xi \\
\leq & \left|B_{\alpha}^{\nu}\right|^{1-\frac{s p}{q}}\left(\int_{B_{\alpha}^{\nu}}\left\|\underline{\kappa}_{\xi} \circ\left(\varphi_{t} * u\right)\right\|_{W^{1, q}\left(Q^{m}\right)}^{q} \mathrm{~d} \xi\right)^{\frac{s p}{q}} \\
\leq & C_{7}\left(\int_{\left\{\left|\varphi_{t} * u-u\right| \geq \alpha\right\}} \int_{B_{\alpha}^{\nu}}\left[1+\left(1+\frac{1}{\operatorname{dist}\left(\varphi_{t} * u(x), X+\xi\right)^{q}}\right)\left|D\left(\varphi_{t} * u\right)(x)\right|^{q}\right] \mathrm{d} \xi \mathrm{d} x\right)^{\frac{s p}{q}} .
\end{aligned}
$$

We have

$$
\begin{aligned}
\int_{B_{\alpha}^{\nu}} \frac{1}{\operatorname{dist}\left(\varphi_{t} * u(x), X+\xi\right)^{q}} \mathrm{~d} \xi & =\int_{B_{\alpha}^{\nu}} \frac{1}{\operatorname{dist}\left(\varphi_{t} * u(x)-X, \xi\right)^{q}} \mathrm{~d} \xi \\
& =\int_{B_{\alpha}^{\nu}+\varphi_{t} * u(x)} \frac{1}{\operatorname{dist}(X, \xi)^{q}} \mathrm{~d} \xi \\
& \leq \int_{B_{R}^{\nu}} \frac{1}{\operatorname{dist}(X, \xi)^{q}} \mathrm{~d} \xi
\end{aligned}
$$

where $R>0$ is such that for every $x \in Q^{m}, B_{\alpha}^{\nu}+\varphi_{t} * u(x) \subset B_{R}^{\nu}$. Since $X$ is a closed subset of a finite union of $\nu-\lfloor s p\rfloor-1$ dimensional planes, assuming in addition that

$$
q<\lfloor s p\rfloor+1,
$$

then the last integral is finite. Thus,

$$
\int_{B_{\alpha}^{\nu}}\left\|\underline{\kappa}_{\xi} \circ\left(\varphi_{t} * u\right)\right\|_{W^{1, q}\left(Q^{m}\right)}^{s p} \mathrm{~d} \xi \leq C_{8}\left(\int_{\left\{\left|\varphi_{t} * u-u\right| \geq \alpha\right\}}\left[1+\left|D\left(\varphi_{t} * u\right)\right|^{q}\right]\right)^{\frac{s p}{q}} .
$$


Inserting this estimate and (2.11) into (2.10), we deduce that

$$
\begin{aligned}
& \int_{B_{\alpha}^{\nu}}\left\|\underline{\kappa}_{\xi} \circ\left(\varphi_{t} * u\right)\right\|_{W^{s, p}\left(Q^{m}\right)}^{p} \mathrm{~d} \xi \\
& \quad \leq C_{9}\left|\left\{\left|\varphi_{t} * u-u\right| \geq \alpha\right\}\right|^{\frac{(1-s) p}{r}}\left(\int_{\left\{\left|\varphi_{t} * u-u\right| \geq \alpha\right\}}\left[1+\left|D\left(\varphi_{t} * u\right)\right|^{q}\right]\right)^{\frac{s p}{q}} .
\end{aligned}
$$

Since $q<p$, by Hölder's inequality and by the identity (2.9) satisfied by the exponents $r, p$ and $q$,

$$
\begin{aligned}
& \int_{B_{\alpha}^{\nu}}\left\|\underline{\kappa}_{\xi} \circ\left(\varphi_{t} * u\right)\right\|_{W^{s, p}\left(Q^{m}\right)}^{p} \mathrm{~d} \xi \\
& \quad \leq C_{10}\left|\left\{\left|\varphi_{t} * u-u\right| \geq \alpha\right\}\right|^{1-s}\left(\int_{\left\{\left|\varphi_{t} * u-u\right| \geq \alpha\right\}}\left[1+\left|D\left(\varphi_{t} * u\right)\right|^{p}\right]\right)^{s} .
\end{aligned}
$$

By the Chebyshev inequality and by Lemma 2.4 ,

$$
\begin{aligned}
\left|\left\{\left|\varphi_{t} * u-u\right| \geq \alpha\right\}\right| & \leq \frac{1}{\alpha^{p}} \int_{\left\{\left|\varphi_{t} * u-u\right| \geq \alpha\right\}}\left|\varphi_{t} * u-u\right|^{p} \\
& \leq C_{11} t^{s p} \int_{\left\{\left|\varphi_{t} * u-u\right| \geq \alpha\right\}}\left(D^{s, p} u\right)^{p} .
\end{aligned}
$$

By Lemma 2.4, we also have

We conclude that

$$
\int_{\left\{\left|\varphi_{t} * u-u\right| \geq \alpha\right\}}\left|D\left(\varphi_{t} * u\right)\right|^{p} \leq \frac{C_{12}}{t^{(1-s) p}} \int_{\left\{\left|\varphi_{t} * u-u\right| \geq \alpha\right\}}\left(D^{s, p} u\right)^{p} .
$$

$$
\int_{B_{\alpha}^{\nu}}\left\|\underline{\kappa}_{\xi} \circ\left(\varphi_{t} * u\right)\right\|_{W^{s, p}\left(Q^{m}\right)}^{p} \mathrm{~d} \xi \leq C_{13}\left(t^{s p}+1\right) \int_{\left\{\left|\varphi_{t} * u-u\right| \geq \alpha\right\}}\left(D^{s, p} u\right)^{p} .
$$

This proves the claim.

\section{Strong Density For $\boldsymbol{s} \boldsymbol{p}<\mathbf{1}$}

The proof of Theorem 2 when $s p<1$ relies on the density of step functions in $W^{s, p}$ based on a Haar projection [4]. This analytical step is developped in Propositions 3.1 and 3.2 below. Then, a standard tool from Differential topology (Proposition 3.3) allows us to reduce the problem to an approximation of a map with values in a convex set and this can be carried out by convolution.

Given a function $v \in L^{1}\left(Q^{m} ; \mathbb{R}^{\nu}\right)$, we consider the Haar projection $E_{j}(v)$ : $Q^{m} \rightarrow \mathbb{R}^{\nu}$ defined almost everywhere on $Q^{m}$. More precisely, denoting by $K_{2^{-j}}^{m}$ the standard cubication of $Q^{m}$ in $2^{j m}$ cubes of radius $2^{-j}$, for every $\sigma \in K_{2^{-j}}^{m}$ the function $E_{j}(v)$ is constant in int $\sigma$ and for $x \in \operatorname{int} \sigma$,

$$
E_{j}(v)(x)=\frac{1}{|\sigma|} \int_{\sigma} v .
$$

In particular, $E_{j}(v)$ is a step function.

Proposition 3.1. Let $v \in L^{p}\left(Q^{m} ; \mathbb{R}^{\nu}\right)$. Then, for every $j \in \mathbb{N}_{*}$,

$$
\left\|E_{j}(v)\right\|_{L^{p}\left(Q^{m}\right)} \leq\|v\|_{L^{p}\left(Q^{m}\right)}
$$

and the sequence $\left(E_{j}(v)\right)_{j \in \mathbb{N}_{*}}$ converges strongly to $v$ in $L^{p}\left(Q^{m} ; \mathbb{R}^{\nu}\right)$. 
Proof. The estimate follows from Hölder's inequality. To prove the convergence of the sequence $\left(E_{j}(v)\right)_{j \in \mathbb{N}_{*}}$, we write

$$
\begin{aligned}
\left\|E_{j}(v)-v\right\|_{L^{p}\left(Q^{m}\right)}^{p} & =\sum_{\sigma \in K_{2^{-j}}^{m}} \int_{\sigma}\left|v(x)-\frac{1}{|\sigma|} \int_{\sigma} v\right|^{p} \mathrm{~d} x \\
& \leq \sum_{\sigma \in K_{2^{-j}}^{m}} \frac{1}{|\sigma|} \int_{\sigma} \int_{\sigma}|v(x)-v(y)|^{p} \mathrm{~d} x \mathrm{~d} y .
\end{aligned}
$$

Approximating $v$ in $L^{p}\left(Q^{m} ; \mathbb{R}^{\nu}\right)$ by a continuous function, we deduce that the righthand side converges to 0 as $j$ tends to infinity. This gives the conclusion.

The counterpart of the previous proposition still holds in the case of fractional Sobolev spaces $W^{s, p}$ for $s p<1$ and is due to Bourgain, Brezis and Mironescu [4, Corollary A.1]:

Proposition 3.2. Let $v \in W^{s, p}\left(Q^{m} ; \mathbb{R}^{\nu}\right)$. If $s p<1$, then for every $j \in \mathbb{N}_{*}$,

$$
\left[E_{j}(v)\right]_{W^{s, p}\left(Q^{m}\right)} \leq C[v]_{W^{s, p}\left(Q^{m}\right)}
$$

for some constant $C>0$ depending on $s, p$ and $m$. In addition, the sequence $\left(E_{j}(v)\right)_{j \in \mathbb{N}_{*}}$ converges strongly to $v$ in $W^{s, p}\left(Q^{m} ; \mathbb{R}^{\nu}\right)$.

The proof of Bourgain, Brezis and Mironescu is based on a characterization of the fractional Sobolev spaces $W^{s, p}$ for $s p<1$ due to Bourdaud [10] in terms of the Haar basis. We present an alternative argument relying directly on the Gagliardo seminorm. The main ingredient is the following:

Claim. If $s p<1$, then for every $\sigma, \rho \in K_{2^{-j}}^{m}$,

$$
\int_{\sigma} \int_{\rho} \frac{1}{|x-y|^{m+s p}} \mathrm{~d} x \mathrm{~d} y \leq C^{\prime} \frac{|\sigma||\rho|}{\delta(\sigma, \rho)^{m+s p}},
$$

where

$$
\delta(\sigma, \rho)=\sup \{|x-y|: x \in \sigma \text { and } y \in \rho\}
$$

and the constant $C^{\prime}>0$ depends on $m$ and $s p$.

Proof of the claim. For every $(x, y) \in \sigma \times \rho$,

$$
|x-y| \geq \delta(\sigma, \rho)-\operatorname{diam} \sigma-\operatorname{diam} \rho=\delta(\sigma, \rho)-2^{-j+2} \sqrt{m} .
$$

If $\delta(\sigma, \rho) \geq 2^{-j+3} \sqrt{m}$, then $\frac{1}{2} \delta(\sigma, \rho) \leq|x-y| \leq \delta(\sigma, \rho)$ and the result follows in this case. Since the indicator function of the unit cube $\chi_{Q^{m}}$ belongs to $W^{s, p}\left(\mathbb{R}^{m}\right)$ for $s p<1$, a scaling argument leads to the following estimate

$$
\frac{1}{|\sigma||\rho|} \int_{\sigma} \int_{\rho} \frac{1}{|x-y|^{m+s p}} \mathrm{~d} x \mathrm{~d} y \leq C_{1} 2^{j(m+s p)} .
$$

In turn, this implies the claim when $\delta(\sigma, \rho)<2^{-j+3} \sqrt{m}$.

Proof of Proposition 3.2. Let $\sigma, \rho \in K_{2^{-j}}^{m}$. For $x \in \sigma$ and $y \in \rho$,

$$
\left|E_{j}(v)(x)-E_{j}(v)(y)\right| \leq \frac{1}{|\sigma||\rho|} \int_{\sigma} \int_{\rho}|v(\tilde{x})-v(\tilde{y})| \mathrm{d} \tilde{x} \mathrm{~d} \tilde{y} .
$$

Thus, by Jensen's inequality,

$$
\left|E_{j}(v)(x)-E_{j}(v)(y)\right|^{p} \leq \frac{1}{|\sigma||\rho|} \int_{\sigma} \int_{\rho}|v(\tilde{x})-v(\tilde{y})|^{p} \mathrm{~d} \tilde{x} \mathrm{~d} \tilde{y} .
$$


We deduce that

$$
\begin{aligned}
\int_{\sigma} \int_{\rho} \frac{\left|E_{j}(v)(x)-E_{j}(v)(y)\right|^{p}}{|x-y|^{m+s p}} \mathrm{~d} x \mathrm{~d} y & \leq \frac{C^{\prime}}{\delta(\sigma, \rho)^{m+s p}} \int_{\sigma} \int_{\rho}|v(\tilde{x})-v(\tilde{y})|^{p} \mathrm{~d} \tilde{x} \mathrm{~d} \tilde{y} \\
& \leq C^{\prime} \int_{\sigma} \int_{\rho} \frac{|v(\tilde{x})-v(\tilde{y})|^{p}}{|x-y|^{m+s p}} \mathrm{~d} \tilde{x} \mathrm{~d} \tilde{y}
\end{aligned}
$$

The desired estimate follows from (3.1) by summation over dyadic cubes in $K_{2^{-j}}^{m}$.

To prove the convergence in $W^{s, p}$ we write for every $\lambda>0$,

$$
\begin{aligned}
& {\left[E_{j}(v)-v\right]_{W^{s, p}\left(Q^{m}\right)}^{p}} \\
& \qquad 2^{p-1} \iint_{D_{\lambda}} \frac{\left|E_{j}(v)(x)-E_{j}(v)(y)\right|^{p}+|v(x)-v(y)|^{p}}{|x-y|^{m+s p}} \mathrm{~d} x \mathrm{~d} y \\
& \quad+\frac{2^{p}\left|Q^{m}\right|}{\lambda^{m+s p}} \int_{Q^{m}}\left|E_{j}(v)-v\right|^{p},
\end{aligned}
$$

where

$$
D_{\lambda}=\left\{(x, y) \in Q^{m} \times Q^{m}:|x-y| \leq \lambda\right\}
$$

By estimate (3.1),

$$
\begin{aligned}
\iint_{D_{\lambda}} \frac{\left|E_{j}(v)(x)-E_{j}(v)(y)\right|^{p}}{|x-y|^{m+s p}} \mathrm{~d} x \mathrm{~d} y & \leq C_{1} \sum_{\substack{\sigma, \rho \in K_{2-j}^{m} \\
(\sigma \times \rho) \cap D_{\lambda} \neq \emptyset}} \int_{\sigma} \int_{\rho} \frac{|v(x)-v(y)|^{p}}{|x-y|^{m+s p}} \mathrm{~d} x \mathrm{~d} y \\
& \leq C_{1} \iint_{D_{\lambda}+Q_{2^{-j+1}}^{2 m}} \frac{|v(x)-v(y)|^{p}}{|x-y|^{m+s p}} \mathrm{~d} x \mathrm{~d} y .
\end{aligned}
$$

Hence,

$$
\begin{aligned}
& {\left[E_{j}(v)-v\right]_{W^{s, p}\left(Q^{m}\right)}^{p}} \\
& \leq C_{2} \iint_{D_{\lambda}+Q_{2^{-j+1}}^{2 m}} \frac{|v(x)-v(y)|^{p}}{|x-y|^{m+s p}} \mathrm{~d} x \mathrm{~d} y+\frac{2^{p}\left|Q^{m}\right|}{\lambda^{m+s p}} \int_{Q^{m}}\left|E_{j}(v)-v\right|^{p} .
\end{aligned}
$$

By Proposition 3.1 the last integral tends to zero as $j$ tends to infinity. Thus,

$$
\limsup _{j \rightarrow \infty}\left[E_{j}(v)-v\right]_{W^{s, p}\left(Q^{m}\right)}^{p} \leq C_{2} \iint_{D_{\lambda}} \frac{|v(x)-v(y)|^{p}}{|x-y|^{m+s p}} \mathrm{~d} x \mathrm{~d} y .
$$

The conclusion follows by choosing $\lambda>0$ small enough.

In the proof of Theorem 2 we need the following property from Differential topology:

Proposition 3.3. Let $N^{n}$ be a connected manifold. Then, for every finite subset $A$ in $N^{n}$, there exists an open neighborhood of $A$ in $N^{n}$ which is diffeomorphic to the Euclidean ball $B^{n}$.

Proof. Let $U \subset N^{n}$ be an open set which is diffeomorphic to the Euclidean ball $B^{n}$. There exists a diffeomorphism $f: N^{n} \rightarrow N^{n}$ mapping $A$ into $U$ [17, Lemma 5.2.6]; in dimension $n \geq 2$ this follows from the multi-transitivity in the group of diffeomorphism of $N^{n}$ [1, Lemma 2.1.10]. The set $f^{-1}(U)$ is thus diffeomorphic to $B^{n}$ and contains $A$. 
Proof of Theorem 2 when $s p<1$. Let $u \in W^{s, p}\left(Q^{m} ; N^{n}\right)$ and let $\iota>0$ be such that the nearest point projection $\Pi$ into $N^{n}$ is smooth on $N^{n}+\bar{B}_{\iota}^{\nu}$.

Let $b \in N^{n}$. For every $j \in \mathbb{N}_{*}$, we define $u_{j}: Q^{m} \rightarrow \mathbb{R}^{\nu}$ for $x \in Q^{m}$ by

$$
u_{j}(x)= \begin{cases}E_{j}(u)(x) & \text { if } \operatorname{dist}\left(E_{j}(u)(x), N^{n}\right)<\iota, \\ b & \text { otherwise. }\end{cases}
$$

Then, $\left(u_{j}\right)_{j \in \mathbb{N}_{*}}$ is a sequence of step functions with values into $N^{n}+B_{\iota}^{\nu}$. By the triangle inequality,

$$
\left\|u_{j}-u\right\|_{W^{s, p}\left(Q^{m}\right)} \leq\left\|E_{j}(u)-u_{j}\right\|_{W^{s, p}\left(Q^{m}\right)}+\left\|E_{j}(u)-u\right\|_{W^{s, p}\left(Q^{m}\right)} .
$$

We need to estimate the first term in the right hand side of this inequality. Since the range of $E_{j}(u)$ is contained in a fixed bounded set - for instance the convex hull of $N^{n}-$, for every $j \in \mathbb{N}_{*}$,

$$
\begin{aligned}
\left\|E_{j}(u)-u_{j}\right\|_{L^{p}\left(Q^{m}\right)} & =\left\|E_{j}(u)-b\right\|_{L^{p}\left(\left\{\operatorname{dist}\left(E_{j}(u), N^{n}\right) \geq \iota\right\}\right)} \\
& \leq C_{1}\left|\left\{x: \operatorname{dist}\left(E_{j}(u)(x), N^{n}\right) \geq \iota\right\}\right|^{\frac{1}{p}} .
\end{aligned}
$$

Since $\left|E_{j}(u)(x)-u(x)\right| \geq \iota$ on $\left\{x: \operatorname{dist}\left(E_{j}(u)(x), N^{n}\right) \geq \iota\right\}$, we get

$$
\left\|E_{j}(u)-u_{j}\right\|_{L^{p}\left(Q^{m}\right)} \leq C_{1}\left|\left\{x:\left|E_{j}(u)(x)-u(x)\right| \geq \iota\right\}\right|^{\frac{1}{p}} .
$$

Thus, by the Chebyshev inequality,

$$
\left\|E_{j}(u)-u_{j}\right\|_{L^{p}\left(Q^{m}\right)} \leq \frac{C_{1}}{\iota^{\frac{1}{p}}}\left\|E_{j}(u)-u\right\|_{L^{p}\left(Q^{m}\right)} .
$$

We need a similar estimate for the Gagliardo seminorm $W^{s, p}$ :

Claim. There exists $C>0$ depending on $s, p$ and $m$ such that for every $j \in \mathbb{N}_{*}$

$$
\left[E_{j}(u)-u_{j}\right]_{W^{s, p}\left(Q^{m}\right)} \leq C\left(\left[E_{j}(u)-u\right]_{W^{s, p}\left(Q^{m}\right)}+[u]_{W^{s, p}\left(A_{j}\right)}\right),
$$

where $A_{j}=\left\{x \in Q^{m}: \operatorname{dist}\left(E_{j}(u)(x), N^{n}\right) \geq \iota\right\}$.

Proof of the claim. First note that

$$
\begin{aligned}
{\left[E_{j}(u)-u_{j}\right]_{W^{s, p}\left(Q^{m}\right)}^{p}=2 \sum_{\sigma \in \mathcal{A}} } & \sum_{\rho \in K_{2^{-j}}^{m} \backslash \mathcal{A}} \int_{\sigma} \int_{\rho} \frac{\left|E_{j}(u)(x)-b\right|^{p}}{|x-y|^{m+s p}} \mathrm{~d} x \mathrm{~d} y \\
& +\sum_{\sigma \in \mathcal{A}} \sum_{\rho \in \mathcal{A}} \int_{\sigma} \int_{\rho} \frac{\left|E_{j}(u)(x)-E_{j}(u)(y)\right|^{p}}{|x-y|^{m+s p}} \mathrm{~d} x \mathrm{~d} y,
\end{aligned}
$$

where

$$
\mathcal{A}=\left\{\sigma \in K_{2^{-j}}^{m}: \operatorname{dist}\left(E_{j}(u)(x), N^{n}\right) \geq \iota \text { for } x \in \sigma\right\} .
$$

By (3.1), we have

$$
\sum_{\sigma \in \mathcal{A}} \sum_{\rho \in \mathcal{A}} \int_{\sigma} \int_{\rho} \frac{\left|E_{j}(u)(x)-E_{j}(u)(y)\right|^{p}}{|x-y|^{m+s p}} \mathrm{~d} x \mathrm{~d} y \leq C_{1}[u]_{W^{s, p}\left(A_{j}\right)}^{p} .
$$

We now estimate the term

$$
I=\sum_{\sigma \in \mathcal{A}} \sum_{\rho \in K_{2^{-j}}^{m} \backslash \mathcal{A}} \int_{\sigma} \int_{\rho} \frac{\left|E_{j}(u)(x)-b\right|^{p}}{|x-y|^{m+s p}} \mathrm{~d} x \mathrm{~d} y .
$$

Since the image of $u$ is contained in $N^{n}$ and $N^{n}$ is bounded, there exists a constant $C_{2}>0$ such that for every $j \in \mathbb{N}_{*}$,

$$
\left|E_{j}(u)-b\right| \leq C_{2} .
$$


Since $s p<1$, by the Claim following Proposition 3.2 ,

$$
\begin{aligned}
I & \leq C_{2}^{p} \sum_{\sigma \in \mathcal{A}} \sum_{\rho \in K_{2^{-j}}^{m} \backslash \mathcal{A}} \int_{\sigma} \int_{\rho} \frac{1}{|x-y|^{m+s p}} \mathrm{~d} x \mathrm{~d} y \\
& \leq C_{3} \sum_{\sigma \in \mathcal{A}} \sum_{\rho \in K_{2^{-j}}^{m} \backslash \mathcal{A}} \frac{|\sigma||\rho|}{\delta(\sigma, \rho)^{m+s p}} .
\end{aligned}
$$

For every $\sigma \in \mathcal{A}$,

$$
\int_{\sigma}\left|E_{j}(u)-u\right|^{p} \geq \iota^{p}|\sigma|
$$

Thus,

$$
I \leq \frac{C_{3}}{\iota^{p}} \sum_{\sigma \in \mathcal{A}} \sum_{\rho \in K_{2^{-j}}^{m} \backslash \mathcal{A}} \frac{|\rho|}{\delta(\sigma, \rho)^{m+s p}} \int_{\sigma}\left|E_{j}(u)-u\right|^{p}
$$

Since $E_{j}(u)=\frac{1}{|\rho|} \int_{\rho} u$ in $\rho$, for $x \in \sigma$ we have by the triangle inequality,

$$
\left|E_{j}(u)(x)-u(x)\right| \leq \frac{1}{|\rho|} \int_{\rho}\left|E_{j}(u)(x)-u(x)-E_{j}(u)(y)+u(y)\right| \mathrm{d} y .
$$

Thus, by Jensen's inequality,

$$
\left|E_{j}(u)(x)-u(x)\right|^{p} \leq \frac{1}{|\rho|} \int_{\rho}\left|E_{j}(u)(x)-u(x)-E_{j}(u)(y)+u(y)\right|^{p} \mathrm{~d} y .
$$

We deduce that

$$
I \leq \frac{C_{3}}{\iota^{p}} \sum_{\sigma \in \mathcal{A}} \sum_{\rho \in K_{2^{-j}}^{m} \backslash \mathcal{A}} \int_{\sigma} \int_{\rho} \frac{\left|E_{j}(u)(x)-u(x)-E_{j}(u)(y)+u(y)\right|^{p}}{|x-y|^{m+s p}} \mathrm{~d} y \mathrm{~d} x
$$

and the claim follows.

By the triangle inequality (3.2), by estimate (3.3) and by the previous claim, we have for every $j \in \mathbb{N}_{*}$,

$$
\left\|u_{j}-u\right\|_{W^{s, p}\left(Q^{m}\right)} \leq C_{4}\left\|E_{j}(u)-u\right\|_{W^{s, p}\left(Q^{m}\right)}+C[u]_{W^{s, p}\left(A_{j}\right)} .
$$

Since $\left(E_{j}(u)\right)_{j \in \mathbb{N}_{*}}$ converges to $u$ in measure and $u(x) \in N^{n}$ for a.e. $x \in Q^{m}$, the sequence $\left(\left|A_{j}\right|\right)_{j \in \mathbb{N}_{*}}$ converges to zero. Since $u \in W^{s, p}\left(Q^{m}\right)$, by the Dominated convergence theorem we get

$$
\lim _{j \rightarrow+\infty}[u]_{W^{s, p}\left(A_{j}\right)}=0 .
$$

Applying Proposition [3.2, we deduce that $\left(u_{j}\right)_{j \in \mathbb{N}_{*}}$ converges strongly to $u$ in $W^{s, p}\left(Q^{m} ; \mathbb{R}^{\nu}\right)$. Since $u_{j}\left(Q^{m}\right) \subset N^{n}+\bar{B}_{\iota}^{\nu}$, the sequence $\left(\Pi \circ u_{j}\right)_{j \in \mathbb{N}_{*}}$ converges strongly to $u$ in $W^{s, p}\left(Q^{m} ; N^{n}\right)$.

To conclude the proof of Theorem [2, we may then assume that $u$ is a step function. In this case, $u\left(Q^{m}\right)$ is a finite set of points in $N^{n}$. By Proposition 3.3, there exists an open neighborhood $U$ of $u\left(Q^{m}\right)$ in $N^{n}$ and a smooth diffeomorhism $\Phi: \bar{U} \rightarrow \bar{B}^{n}$. Since the set $\bar{B}^{n}$ is convex, there exists a sequence of smooth maps $\left(v_{i}\right)_{i \in \mathbb{N}}$ in $C^{\infty}\left(\bar{Q}^{m} ; \bar{B}^{n}\right)$ which converges strongly to $\Phi \circ u$ in $W^{s, p}\left(Q^{m} ; \bar{B}^{n}\right)$. Hence, the sequence $\left(\Phi^{-1} \circ v_{i}\right)_{i \in \mathbb{N}}$ converges strongly to $u$ in $W^{s, p}\left(Q^{m} ; N^{n}\right)$. This completes the proof of Theorem 2 for $s p<1$.

Acknowledgments. The second (ACP) and third (JVS) authors were supported by the Fonds de la Recherche scientifique-FNRS. 


\section{REFERENCES}

[1] A. Banyaga, The structure of classical diffeomorphism groups, Mathematics and its Applications, vol. 400, Kluwer Academic Publishers Group, Dordrecht, 1997. $\uparrow 13$

[2] F. Bethuel, The approximation problem for Sobolev maps between two manifolds, Acta Math. 167 (1991), 153-206. $\uparrow 2,3$

[3] _ Approximations in trace spaces defined between manifolds, Nonlinear Anal. 24 (1995), 121-130. $\uparrow 2$

[4] J. Bourgain, H. Brezis, and P. Mironescu, Lifting in Sobolev spaces, J. Anal. Math. 80 (2000), 37-86. $\uparrow 2,3,11,12$

[5] - $H^{1 / 2}$ maps with values into the circle: minimal connections, lifting, and the Ginzburg-Landau equation, Publ. Math. Inst. Hautes Études Sci. (2004), 1-115. ^2, 3, 6,

[6] P. Bousquet, A. C. Ponce, and J. Van Schaftingen, Density of smooth maps for fractional Sobolev spaces $W^{s, p}$ into $\ell$ simply connected manifolds when $s \geq 1$, Confluentes Math. 5 (2013), 3-22. $\uparrow 2$

[7] _ Strong density for higher order Sobolev spaces into compact manifolds, submitted paper. $\uparrow 3$

[8] H. Brezis and P. Mironescu, Gagliardo-Nirenberg, composition and products in fractional Sobolev spaces, J. Evol. Equ. 1 (2001), 387-404. $\uparrow 3$

[9] H. Brezis and L. Nirenberg, Degree theory and BMO. I. Compact manifolds without boundaries, Selecta Math. (N.S.) 1 (1995), 197-263. 11,2

[10] G. Bourdaud, Ondelettes et espaces de Besov, Rev. Mat. Iberoamericana 11 (1995), 477-511. $\uparrow 12$

[11] M. Escobedo, Some remarks on the density of regular mappings in Sobolev classes of $S^{M}$ valued functions, Rev. Mat. Univ. Complut. Madrid 1 (1988), 127-144. $\uparrow 2$

[12] E. Gagliardo, Caratterizzazioni delle tracce sulla frontiera relative ad alcune classi di funzioni in $n$ variabili, Rend. Sem. Mat. Univ. Padova 27 (1957), 284-305. 11

[13] P. Hajłasz, Approximation of Sobolev mappings, Nonlinear Anal. 22 (1994), 1579-1591. $\uparrow 2$

[14] F. Hang and F. Lin, Topology of Sobolev mappings. II, Acta Math. 191 (2003), 55-107. 2

[15] R. Hardt and F.-H. Lin, Mappings minimizing the $L^{p}$ norm of the gradient, Comm. Pure Appl. Math. 40 (1987), 555-588. $\uparrow 2,3$

[16] A. Hatcher, Algebraic topology, Cambridge University Press, Cambridge, 2002. $\uparrow 3$

[17] M. W. Hirsch, Differential topology, Graduate Texts in Mathematics, vol. 33, Springer-Verlag, New York, 1994. $\uparrow 13$

[18] M. Marcus and V. J. Mizel, Every superposition operator mapping one Sobolev space into another is continuous, J. Funct. Anal. 33 (1979), 217-229. $\uparrow 6$

[19] V. Maz'ya and T. Shaposhnikova, An elementary proof of the Brezis and Mironescu theorem on the composition operator in fractional Sobolev spaces, J. Evol. Equ. 2 (2002), 113-125. $\uparrow 2$

[20] _ On the Brezis and Mironescu conjecture concerning a Gagliardo-Nirenberg inequality for fractional Sobolev norms, J. Math. Pures Appl. (9) 81 (2002), 877-884. ^3, 5

[21] P. Mironescu, On some properties of $S^{1}$-valued fractional Sobolev spaces, Noncompact problems at the intersection of geometry, analysis, and topology, Contemp. Math., vol. 350, Amer. Math. Soc., Providence, RI, 2004, pp. 201-207. $\uparrow 2$

[22] __ Sobolev maps on manifolds: degree, approximation, lifting, Perspectives in nonlinear partial differential equations, Contemp. Math., vol. 446, Amer. Math. Soc., Providence, RI, 2007, pp. 413-436. $\uparrow 2$

[23] D. Mucci, Strong density results in trace spaces of maps between manifolds, Manuscripta Math. 128 (2009), 421-441. $\uparrow 2$

[24] T. Rivière, Dense subsets of $H^{1 / 2}\left(S^{2}, S^{1}\right)$, Ann. Global Anal. Geom. 18 (2000), 517-528. $\uparrow 3$

[25] R. Schoen and K. Uhlenbeck, Boundary regularity and the Dirichlet problem for harmonic maps, J. Differential Geom. 18 (1983), 253-268. $\uparrow 2$

[26] J. W. Vick, Homology theory: An introduction to algebraic topology, 2nd ed., Graduate Texts in Mathematics, vol. 145, Springer-Verlag, New York, 1994. $\uparrow 4$ 
Aix-Marseille Université, Laboratoire d'analyse, topologie, probabilités UMR7353, 39 rue Frédéric Joliot Curie, 13453 Marseille Cedex 13, France

E-mail address: Pierre.Bousquet@univ-amu.fr

Université Catholique de Louvain, Institut de Recherche en Mathématique et Physique, Chemin du cyclotron 2, Bte L7.01.02, 1348 Louvain-la-Neuve, Belgium E-mail address: Augusto.Ponce@uclouvain.be

Université CAtholique de Louvain, Institut de Recherche en Mathématique et Physique, Chemin du cyclotron 2, Bte L7.01.02, 1348 Louvain-la-Neuve, Belgium

E-mail address: Jean.VanSchaftingen@uclouvain.be 